\title{
Assessment of the Effects of Facial Hair Removal Laser on Thyroid Hormones
}

\author{
SHEREEN ISMAIL HAJEE, J. H. FATTAH ${ }^{1 *}$, F. F. HASSAN AND N. K. ABDULKAREEM \\ Unit of Biophysics, Department of Basic Science, ${ }^{1}$ Department of Plastic Surgery, College of Medicine, Hawler Medical \\ University, Erbil 44001, Iraq
}

Hajee et al.: Effects of Facial Hair Removal Laser on Thyroid Hormones

\begin{abstract}
Laser hair removal and facial hair removal are popular methods all over the world today. On the other hand, the use of this treatment can have harmful effects on human health because hair growth is closely related to the activity of the thyroid gland and this gland has a significant role in this mechanism. In this regard, the present study was conducted to investigate the effect of laser hair treatment on thyroid function. The present study is a cross-sectional analytical study performed in a private Aesthetic and hair clinic. Seventy women were randomly selected for facial hair removal by two different methods of the diode laser system $(n=140)$ and Alexandrite laser system $(n=140)$ were included in the study. 7 cc of venous blood samples were taken from all patients before and at the end of laser treatment and after serum isolation in the laboratory, the levels of triiodothyronine, tetraiodothyronine and thyroid stimulating hormone, hemoglobin and total bilirubin were examined. In our study, 280 women in two groups of 140 patients were evaluated. The mean age of women was $35.24 \pm 12.5$ in the diode group and $35.49 \pm 12.8$ in the Alexandrite group. Our results showed that the levels of triiodothyronine, tetraiodothyronine and thyroid stimulating hormones before laser treatment were not statistically different in the study groups and the concentration of thyroid hormones in each of the study groups before and after laser treatment was not statistically significant. There were no significant changes in mean hemoglobin and total bilirubin levels before and after laser therapy $(p>0.05)$. Also, there were no significant differences among the groups of Diode or Alexandrite lasers in case of hemoglobin and total bilirubin $(p>0.05)$. Based on the results of the present study, we concluded that the use of diode and alexandrite laser systems does not affect the concentration of thyroid hormones or hemoglobin and bilirubin.
\end{abstract}

Key words: Thyroid hormones, diode laser system, alexandrite laser system, facial hair

Since the last century the laser occupied a large degree of attention in the scientific and technological field's invention of laser causes a chain of important changes in science development, industrial, commercial, agricultural, specification and measurement, cosmetic surgery and medical therapy ${ }^{[1,2]}$. It had been used for therapy that improves tissue repair (skin wounds, muscle, tendon, bone, nerves), reduces inflammation and reduces pain wherever the beam is applied. Usually applied by a doctor, therapist or technician, treatments typically take 1-10 min and should be applied two or more times a $\mathrm{w}^{[3-6]}$. Although, laser irradiation is now being considered a therapy of choice for many difficult pain management challenges and is becoming a common practice in medicine there is no clear understanding of its side effects from one side and its physiological action on endocrine gland from other side. Moreover, the available literature lacks the effect of laser on thyroid gland directly and their physiological changes in the body, since the thyroid is a superficial gland, and a laser can easily access it transcutaneous. Thus, this experiment designed to study the effect of laser therapy on thyroid hormones and other blood parameters which could be affected by laser therapy as well as hemoglobin and total bilirubin in treated persons with hair removal laser. This study is a descriptiveanalytical study that was performed with the aim of measuring the levels of Thyroxine (T4), Thyroid Stimulating Hormone (TSH) and Triiodothyronine (T3) in females referring to Aesthetic and hair clinic to

*Address for correspondence

E-mail: jalal.hamasaih@hmu.edu.krd 
remove facial hair. The statistical population includes 280 females who referred to the aesthetic and hair clinic to remove facial hair. The sampling method of this study was stratified random. Inclusion criteria included: the presence of unwanted facial hair, Fitzpatrick skin types from 2-4 and adult patients from $21 \mathrm{y}$ to $60 \mathrm{y}$ and complete consent to participate in the study. Exclusion criteria: history of thyroid disease (hyperthyroidism or hypothyroidism, goiter, cancer, autoimmune diseases, skin disease, heart disease, kidney, liver, lung and other systemic diseases (endocrine and non-endocrine), pregnancy, history of radiation, use of drugs affecting thyroid function (amiodarone, contrast agent, anticonvulsant, dopamine and agonists dopamine and dobutamine, steroids, growth hormone and interleukin. A total of 70 females were studied in this study. In order to check and sample the serum, according to the guide that was presented to the females from the previous week, the subjects were given a full explanation about the method of implementing the plan so that they could participate in this plan if they were satisfied. Participants signed written consent forms with full knowledge of the study before being used for the study. And if they did not want to cooperate, they withdrew from the study. In the present study, two laser hair removal systems were used. The facial hair laser was performed under the supervision of a plastic surgeon and in full compliance with the laser protocol. The facial hair laser procedure was performed in $6 \mathrm{w}$ periods in each group and patients whose laser area under the chin was exposed to laser radiation were examined. The high penetration rate of the diode laser with a wavelength of $808 \mathrm{~nm}$ and also the amount of melanin absorption at this wavelength, makes this laser one of the most suitable choices for removing excess hair ${ }^{[7]}$. During laser treatments, proper cooling systems play an effective role in lowering the temperature of the epidermis and increase the speed of healing while reducing the risk of heart damage. The diode laser uses the contact cooling system in the handpiece to allow the skin to cool to sub-zero temperatures during treatment, thus allowing higher laser powers to be applied without burns and pain. The long-pulse alexandrite laser (PhotoGenica LPIR $755 \mathrm{~nm}$; Cynosure Inc, Chelmsford, MA) is based on the principle of thermokinetic selection and targets the melanin in the hair follicle. In this way, the epidermis can be cooled while the melanin in the hair follicles is heated. Cohesive Medicine (Santa Clara, California) and Palomar (Lexington, MA) have introduced LightSheer, an 800-nanometer diode laser with a pulse of up to $30 \mathrm{~ms}^{[8]}$. This technology minimizes the size of the laser by replacing the laser tube with solid state diode circuits. The alexandrite laser is a type of body hair laser that emits a high-energy beam of light from inside an alexandrite crystal. When the laser passes through the alexandrite crystal, it produces a beam of light with a wavelength of $755 \mathrm{~nm}$. The color of the laser light is red. The Alex laser can be set to Q-switch mode, which produces an energetic beam of light with short pulses. This feature of laser hair removal causes the removal of excess hair. The laser wavelength used in this system is $755 \mathrm{~nm}$, which is located in the red, infrared part of the light spectrum. This laser wavelength is similar to a ruby laser, which is mainly absorbed by melanin and hair follicles (instead of the surrounding tissues) ${ }^{[9]}$. In this study, two stages of blood sampling were performed, which was first reported to individuals. After filling in the personal information questionnaire and examination by an endocrinologist, before starting the laser treatment, a $7 \mathrm{cc}$ serum sample was taken from each person to perform T4, TSH and T3 tests. The samples were transferred to the laboratory while maintaining a cold cycle. Information about the variables in this study included thyroid function tests performed by venous blood tests using an autoanalyzer. Information on T4, TSH and T3 concentrations was recorded and stored before the start of the $6 \mathrm{w}$ treatment period. At the end of the 6th w, $7 \mathrm{cc}$ of venous blood was taken from all participants for the second time after the end of the laser treatment period in each group and its serum was transferred to the laboratory and analysed by maintaining a cold cycle. Flow cytometry was used to evaluate subgroups of white blood cells in this study. This system is able to detect a small amount of white blood cells based on their cell markers in different samples and determine their percentage. As mentioned, blood samples were isolated from blood bags before and after filtration to determine the subgroups of white blood cells by flow cytometry and transferred to flow cytometry. To determine the percentage of different subgroups of white blood cells using flow cytometry. In general, the steps described below were performed for samples before and after filtration. After numbering the tubes, $5 \mu \mathrm{l}$ of the corresponding monoclonal antibodies were added to each tube of the sample before filtration and then $100 \mu \mathrm{l}$ of the test sample was added to each tube. In the case of samples after filtration, due to the fact that the number of white blood cells in the samples after filtration shows a sharp decrease, for a more accurate assessment of their number, $5 \mu \mathrm{l}$ of monoclonal antibody and $200 \mu \mathrm{l}$ of sample was added to each of the tubes and thus the volume of blood samples after 
filtration in each test tube was twice the volume of samples before filtration. The tubes were mixed well and placed at $4^{\circ}$ for $30 \mathrm{~min}$. After incubation, the tubes were removed at the desired temperature and then using a Q-prep device for pre-filtered samples, $560 \mu \mathrm{l}$ of formic acid solution for lysis of red blood cells and 260 $\mu l$ of Phosphate Buffer Solution (PBS). To neutralize the long-term effect of the lysis solution, $100 \mu \mathrm{l}$ of $1 \%$ paraformaldehyde solution was added to the tubes to fix the cells and mixed well by vortex. For the samples after filtration (filtered), the volume of added solutions included $1120 \mu \mathrm{l}$ of formic acid solution, $520 \mu \mathrm{l}$ of PBS and $200 \mu \mathrm{l}$ of paraformaldehyde solution respectively. At this stage, the samples were ready for analysis by flow cytometry. The flow cytometer used was the Partec Platelet Additive Solution III (PAS III) model (made in Germany), which is available in the flow cytometry department of the Blood Transfusion Organization. In addition to determining the cell percentage, this device is also able to count absolute white blood cells so that the volume is 0 . Analyze $0.5 \mathrm{ml}$ of the prepared suspension and calculate the number of cells conjugated to fluorescence in this volume and finally present it in the form of Count per ml. After testing the samples with this device, cell fluorescent events were determined. Bilirubin levels were assessed by spectrophotometry in the laboratory. In Jaffe method, creatinine reacts with alkaline medium in the body to form a yellow-orange complex. In the formed color complex, the amount of light absorption is measured at a wavelength of $510 \mathrm{~nm}$. The Berthelot method is based on the urease reaction and the ammonium assay using phenol and hypochlorite. The blue endophenol product will be the measuring frame and the amount of light absorption will be measured at $560 \mathrm{~nm}$. For measuring T4, TSH, T3 from (Roche Diagnostics GmbH, Mannheim, Germany). The normal range for T3 defined for this kit is $0.6-2.1 \mathrm{mIU} / 1$ and for T4 and TSH it was 4.7-12.5 mIU/1 and 0.32-5.2 $\mathrm{mIU} / 1$ respectively. Total bilirubin and hemoglobin were also assessed by laboratory. Analysis of Variance (ANOVA) statistical test for normal data and nonparametric Man-u withney and Kurskal wallis tests were used to compare continuous quantitative variables. Chi square test was used to compare discrete data between different groups. A significance level of less than 0.05 was considered statistically significant. SPSS software version 23 was used for statistical analysis. In the present study, out of 280 participants, 140 were in the Diode laser receiving group and 140 were in the Alexandrite laser receiving group. The mean age of participants in the group receiving diode was $35.24 \pm 12.5$ and in the group receiving alexandrite laser was $35.49 \pm 12.8$ and no statistically significant difference was observed in terms of mean age in the study groups ( $>0.05$ ). At the beginning of the study, we examined the levels of thyroid hormones in the study groups. As expected, all subjects were normal in terms of thyroid hormone levels and no patients in the study had thyroid disorders. There was no statistically significant difference between thyroid hormone levels in the study groups before the intervention $(p>0.05)$. The results of our study on the level of thyroid hormones after laser facial hair in the study groups can be seen in Table 1. After laser treatment, no significant change in serum levels of any thyroid hormones was observed ( $p>0.05$ ) (Table 2). Finally, we examined the changes in thyroid hormones in the study groups separately to understand how each laser hair removal system individually affects thyroid hormones. Our results in this regard did not show a statistically significant difference. In other words, the levels of none of the thyroid hormones in the group receiving diode laser and the group receiving laser before and after the intervention did not show any statistically significant difference $(\mathrm{p}>0.05)$ (Table 1$)$. The results of the study of blood cell count as well as the level of Blood Urea Nitrogen (BUN) and creatinine of the patients can be seen in Table 1. Our results There was no statistically significant difference between white blood cells, red blood cells, BUN and creatinine levels in the pre and post laser study groups $(\mathrm{p}>0.05)$. In other words, the level of white and red blood cells before and after the study did not change in the study groups, also BUN and creatinine in the studied patients did not change much after the laser. There was no statistically significant difference between white blood cells, red blood cells, BUN and creatinine levels in the before and after intervention in study groups $(\mathrm{p}>0.05)$. In other words, the level of white and red blood cells before and after the study did not change in the study groupa and BUN and creatinine did not show much change in the patients after the laser treatment. In the continuation of the study, we examined the percentage of white blood cells of lymphocytes, monocytes, heterophils, eosinophils and basophils. Our results in this regard did not show any statistically significant difference between the study groups and also before and after the study $(p>0.05)$. As shown in fig. 1 there were no significant changes in mean hemoglobin and total bilirubin levels before and after laser therapy $(\mathrm{p}>0.05)$. Also, there were no significant differences among the groups of Diode or Alexandrite lasers ( $>0.05)$. In the present study, we for the first time investigated the effect of 
laser therapy for facial hair removal on thyroid hormone concentration and thyroid function in women. The results of the study did not show any significant relationship between the use of laser and thyroid hormones T3, TSH and T4. To date, studies have been conducted to investigate the relationship between thyroid hormone levels and the effect of the concentration of this hormone on the efficiency of laser treatment to remove excess hair but no study has been conducted to investigate the effect of laser use on thyroid hormone levels ${ }^{[10,11]}$. Laser hair removal, as the most common method of hair removal in the world, is facing many questions today that examine the safety and health of this method and the extent of its harms and risks. A number of these questions are asked by people with thyroid disorders and concerns about the effects of laser hair removal on the condition of their thyroid gland and the likelihood of their medical condition deteriorating. The results of the study reliably answered the questions related to safety and the extent of damage and side effects of this method. In our study, it was found that the use of laser therapy to remove facial hair has no effect on the concentration of T4, T3 and TSH hormones. It should be noted that the laser hair removal process only targets hair follicles and hair pigments and does not even deal with skin tissue ${ }^{[12]}$. This means that laser hair removal is one of the most accurate and targeted methods of hair removal, the destructive function of which is done only for the purpose of destroying hair strands and provided the accuracy and skill in performing the laser hair removal method will not even damage the skin tissue ${ }^{[13]}$. Due to this point, we find that the laser beam in the laser hair removal method does not have the power to penetrate even the tissues under the skin and the penetration of the laser beam into tissues from deep in the body, such as the thyroid gland, is practically impossible ${ }^{[14]}$. The results of the study showed that the use of diode and alexandrite laser systems to remove facial hair in women are safe methods in terms of affecting thyroid function, because these laser systems have no effect on the concentration of thyroid hormones T3, T4 and TSH.

TABLE 1: EVALUATION OF WHITE BLOOD CELLS, RED BLOOD CELLS, BUN AND CREATININE LEVELS

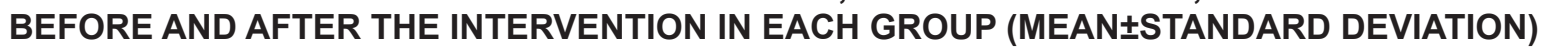

\begin{tabular}{lcccccccc}
\hline & \multicolumn{2}{c}{ Total } & \multicolumn{2}{c}{ Diode group } & \multicolumn{2}{c}{ Alexandrite group } & \multirow{2}{*}{$\mathrm{p}^{*}$} & \multirow{2}{*}{$\mathrm{p}^{* *}$} \\
\cline { 2 - 7 } & Before & After & Before & After & Before & After & & \\
\hline WBC $(103 / \mu \mathrm{l})$ & $5 / 72 \pm 2 / 50$ & $5 / 71 \pm 2 / 38$ & $5 / 65 \pm 2 / 34$ & $5 / 72 \pm 2 / 39$ & $5 / 78 \pm 2 / 65$ & $5 / 69 \pm 2 / 36$ & $0 / 633$ & $0 / 638$ \\
$\mathrm{RBC}(106 / \mu \mathrm{l})$ & $5 / 25 \pm 2 / 09$ & $5 / 30 \pm 2 / 11$ & $5 / 1 \pm 1 / 96$ & $5 / 4 \pm 1 / 88$ & $5 / 4 \pm 2 / 21$ & $5 / 2 \pm 2 / 34$ & $0 / 895$ & $0 / 712$ \\
$\mathrm{Hb}(\mathrm{g} / \mathrm{dl})$ & $10 / 12 \pm 1 / 85$ & $10 / 55 \pm 1 / 7$ & $10 \pm 1 / 9$ & $10 / 23 \pm 1 / 8$ & $10 / 5 \pm 1 / 9$ & $10 / 6 \pm 1 / 6$ & $0 / 365$ & $0 / 245$ \\
$\mathrm{HTC}(\%)$ & $33 / 01 \pm 3 / 40$ & $33 / 54 \pm 3 / 75$ & $32 / 2 \pm 3 / 1$ & $32 / 94 \pm 3 / 6$ & $33 / 81 \pm 3 / 7$ & $34 / 14 \pm 3 / 9$ & $0 / 354$ & $0 / 412$ \\
Lymphocytes (\%) & $40 / 64 \pm 20 / 38$ & $40 / 27 \pm 20 / 47$ & $39 / 65 \pm 19 / 32$ & $38 / 75 \pm 19 / 29$ & $41 / 62 \pm 21 / 43$ & $41 / 79 \pm 21 / 65$ & $0 / 471$ & $0 / 391$ \\
Monocytes (\%) & $0 / 84 \pm 1 / 16$ & $0 / 85 \pm 1 / 24$ & $0 / 85 \pm 1 / 31$ & $0 / 86 \pm 1 / 29$ & $0 / 83 \pm 1 / 01$ & $0 / 84 \pm 1 / 19$ & $0 / 301$ & $0 / 223$ \\
Heterophils (\%) & $61 / 78 \pm 22 / 35$ & $62 / 02 \pm 21 / 94$ & $61 / 44 \pm 22 / 32$ & $61 / 89 \pm 21 / 95$ & $62 / 12 \pm 22 / 37$ & $62 / 14 \pm 21 / 92$ & $0 / 741$ & $0 / 127$ \\
Eosinophils (\%) & $0 / 29 \pm 0 / 66$ & $0 / 29 \pm 0 / 68$ & $0 / 23 \pm 0 / 61$ & $0 / 22 \pm 0 / 62$ & $0 / 35 \pm 0 / 71$ & $0 / 36 \pm 0 / 74$ & $0 / 854$ & $0 / 695$ \\
Basophils (\%) & $0 / 60 \pm 0 / 68$ & $0 / 62 \pm 0 / 69$ & $0 / 58 \pm 0 / 62$ & $0 / 6 \pm 0 / 67$ & $0 / 61 \pm 0 / 74$ & $0 / 63 \pm 0 / 71$ & $0 / 314$ & $0 / 097$ \\
BUN (mg/dl) & $12 / 95 \pm 8 / 92$ & $13 / 14 \pm 8 / 97$ & $12 / 65 \pm 8 / 42$ & $12 / 72 \pm 8 / 55$ & $13 / 24 \pm 9 / 42$ & $13 / 55 \pm 9 / 39$ & $0 / 855$ & $0 / 142$ \\
Creatinine (mg/dl) & $0 / 50 \pm 0 / 20$ & $0 / 52 \pm 0 / 23$ & $0 / 49 \pm 0 / 21$ & $0 / 50 \pm 0 / 26$ & $0 / 51 \pm 0 / 19$ & $0 / 54 \pm 0 / 20$ & $0 / 629$ & $0 / 541$ \\
\hline
\end{tabular}

Note: *Comparison results before and after laser in each group, **Results of comparison before and after in the study groups in totally

TABLE 2: EVALUATION OF TSH, T3 AND T4 LEVELS BEFORE AND AFTER THE INTERVENTION IN EACH GROUP

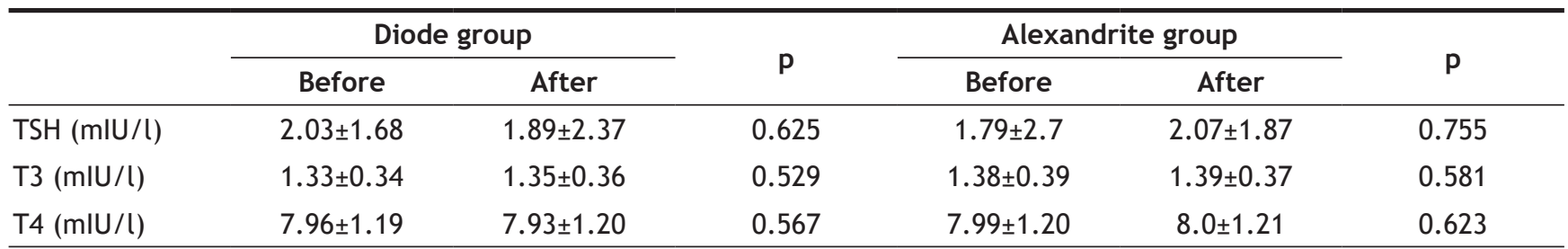




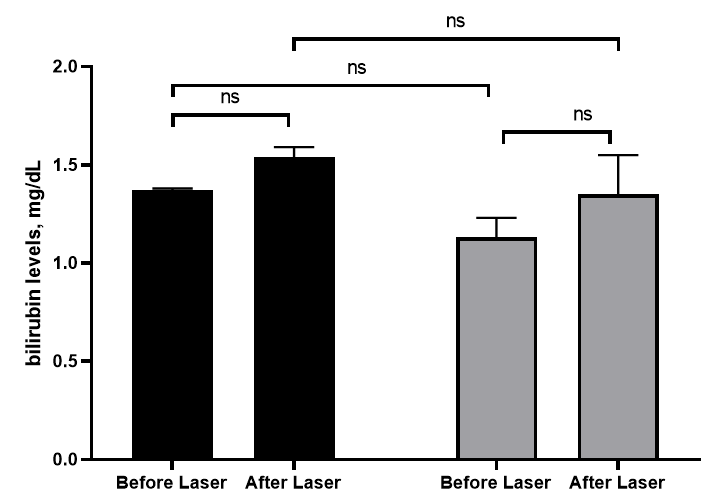

Fig 1: Total bilirubin levels in study groups

\section{Conflict of interest:}

All authors declare that they have no conflict of interest.

\section{REFERENCES}

1. Brosseau L, Welch V, Wells GA, de Bie R, Gam A, Harman K, Morin M, Shea B, Tugwell P. Low level laser therapy (Classes I, II and III) for treating rheumatoid arthritis. Cochrane Database Syst Rev 2005;2005(4):CD002049.

2. Bjordal JM, Couppe C, Chow RT, Tunér J, Ljunggren EA. A systematic review of low level laser therapy with locationspecific doses for pain from chronic joint disorders. Aust J Physiother 2003;49(2):107-16.

3. Huang YY, Chen ACH, Carroll JD, Hamblin MR. Biphasic dose response in low level light therapy. Dose Response 2009;7(4):358-83.

4. Lievens P, Van Der Veen P. Woundhealing process: Influence of LLLT on the proliferation of fibroblasts and on the lymphatic regeneration. InBook of Abstracts of the VIIth International Congress of the European Medical Laser Association 2000;323.

5. AL-Mustawfi NS. The physiological effect of laser therapy on normal and hyperthyroid female rabbits. Baghdad Univ; 2009.

6. FR Mohammed I, S Al-Azawi T, S AL-Mustawfi N. Effect of laser treatment on thyroid gland hormones in female rabbits. Iraqi J Vet Sci 2011;25(2):61-4.

7. Raj Kirit EP, Sivuni A, Ponugupati S, Gold MH. Efficacy and safety of triple wavelength laser hair reduction in skin types IV to V. J Cosmet Dermatol 2021;20(4):1117-23.

8. Bonan P, Troiano M, Verdelli A. Safety and efficacy of single pass vs. multipass emission with 755 alexandrite laser for all-skin-type hair removal: A pilot study. Dermatol Ther 2020;33(6):e14001.
9. Dorgham NA, Dorgham DA. Lasers for reduction of unwanted hair in skin of colour: a systematic review and meta-analysis. J Eur Acad Dermatology Venereol 2020;34(5):948-55.

10. Carter EE, Saade DS, Vashi NA. Presurgical Laser Hair Removal: Protocoling a Safe and Effective Procedure for Transgender Patients. Transgend Health 2021;6(4):201-6.

11. Kuo SC, Huang F, Chi SY, Lin HP, Chien PC, Hsieh CH. Investigate the improvement of facial skin texture with the VISIA system after total thyroidectomy. BMC Surg 2021;21(1):1-6.

12. Durante C, Grani G, Lamartina L, Filetti S, Mandel SJ, Cooper DS. The diagnosis and management of thyroid nodules: a review. JAMA 2018;319(9):914-24.

13. Asiri MS, Alharbi M, Alkadi T, Abouammoh M, Al-Amry M, ALZahrani Y, et al. Ocular injuries secondary to alexandrite laser-assisted hair removal. Can J Ophthalmol 2017;52(2):e71-5.

14. Crowell EL, Jampel H, Berkenstock M. Alexandrite laser induced uveitis \& pigment dispersion: A case report and review of the literature. Am J Ophthalmol Case Rep 2020;18:100632.

This is an open access article distributed under the terms of the Creative Commons Attribution-NonCommercial-ShareAlike 3.0 License, which allows others to remix, tweak, and build upon the work non-commercially, as long as the author is credited and the new creations are licensed under the identical terms

This article was originally published in a special issue, "Diagnostic and Therapeutic Advances in Biomedical Research and Pharmaceutical Sciences"

Indian J Pharm Sci 2021:83(5)Spl Issue "283-287" 